\title{
Clinicopathological Profile and Outcome of Dengue Fever: A Tertiary Care Hospital Experience
}

\author{
MA RAHMAN $^{\mathrm{a}}$, MUH BEGUM $^{\mathrm{b}}$, HMN UDDIN $^{\mathrm{c}}, \mathrm{S}_{\text {MONIRA }}^{\mathrm{d}}$, MB MILLAT
}

\begin{abstract}
:
Introduction: Diverse manifestation of recent dengue outbreak has posed a challenge to pre-existing nature of dengue virus infection and management. This study was designed to observe clinicopathological profile and analyze the diverse presentation and outcome of dengue syndrome in recent outbreak in Bangladesh.
\end{abstract}

Methods: This was a hospital based observational study, carried out in Medicine department of Combined Military Hospital (CMH) Dhaka cantonment in between May and September 2019. Total 300 laboratory-confirmed dengue cases aged more than 11 years presenting within 7 days of symptom onset were studied. Patients who presented 7 days after the onset of symptoms or those who were transferred to other hospitals were excluded from study.

Results: Total patients were 300 with male predominance $(187,62.3 \%)$ and mean age $\pm S D$ was $37.6 \pm 7.5 y e a r s$ with age range 12 to 76 years; common presentations were fever (300, $100 \%)$,headache $(265,88.3 \%)$, skin rash (197, 65.7\%), bodyache (186, 62.0\%),vomiting (152, 50.7\%), diarrhoea $(65,21.7 \%)$, abdominal pain $(58,19.3 \%)$, and bleeding manifestation (36, 12.0). Eighty four (28\%) patient had classical dengue fever (DF), 61 (20.3\%) had dengue

Introduction:

Dengue virus is an arthropod borne virus of genus flavivirus belonging to family Flaviviridae. It is a singlestranded RNA virus. There are four genetically related but antigenically distinct Dengue virus (DENV) serotypes (DENV-1, DENV-2, DENV-3, and DENV-4). A haemorrhagic fever (DHF), 45(15\%) had dengue shock syndrome (DSS) and 110(36.7\%) had expanded dengue syndrome(EDS). Relevant investigations showed 157(52.3\%) patients had leukopenia,18(6\%) had leukocytosis, 254(84.7\%) had thrombocytopenia with lowest platelet count $1 \times 10^{9} / \mathrm{L}, 135(45 \%)$ had abnormal ALT, 110(36.7\%) had abnormal AST, 84(28\%) had hyponatraemia,43(14.3\%) had AKI,125(41.7\%) had pleural effusion with 36(12\%) bilateral;102(34\%) had ascites, 25(8.3\%) developed acalculous cholecystitis;7(2.3\%) patient developed cerebrovascular accident. Eighteen (6\%) patients required mechanical ventilation and $15(5 \%)$ patients required haemodialysis. Regarding outcome, 5 (1.7\%) patients died and 295 (98.3\%) patients survived.

Conclusion: Dengue fever was presented with common as well as other features and involved a number of organs including liver, kidneys, brain, pleura, peritoneum, and gall bladder and had diverse manifestations and adverse outcome.

Key Words: Dengue fever, Expanded dengue syndrome, Plasma leakage, Acalculous cholecystitis, Acute pancreatitis, Acute kidney injury.

(J Bangladesh Coll Phys Surg 2021; 39: 213-219) DOI: https://doi.org/10.3329/jbcps.v39i4.55941

recently published article has unveiled a new serotype (DENV-5), to be added to the existing ones ${ }^{1}$. Dengue viral infections are one of the most important mosquitoborne diseases in the world. It is endemic in over 100 countries $^{2}$. About $40 \%$ of people all over the world live in countries where dengue is endemic. A vast majority

a. Lt. Col (Dr.) Md. Anisur Rahman, OJT in Interventional Gastroenterology (India), Classified Medicine Specialist and Gastroenterologist, Dept. of Gastroenterology Combined Military Hospital (CMH) Dhaka Cantonment. Mobile: 01521-248085, Email: arafanis@gmail.com

b. Dr. Most. Umme Habiba Begum, Associate Professor, Dept. of Paediatrics, Rangpur Community Medical College, Rangpur.

c. Maj. (Dr.) Hafez Md Nizam Uddin, Graded Specialist in Medicine, Dept. of Medicine, Combined Military Hospital (CMH) Dhaka Cantonment.

d. Maj. (Dr.) Serazum Monira, Armed Forces Medical Institute (AFMI) Dhaka Cantonment.

e. Dr Md Bodrul Millat, Lecturer, Dept. of Biochemistry Rangpur Army Medical College, Rangpur.

Address of Correspondence: Lt. Col. (Dr.) Md Anisur Rahman MBBS, FCPS (Medicine), OJT in Interventional Gastroenterology (India), Classified Medicine Specialist and Gastroenterologist, Dept. of Gastroenterology Combined Military Hospital (CMH) Dhaka Cantonment. Mobile: 01521-248085,

Email: arafanis@gmail.com

Revive: 3 October, 2019

Accept: 18 May, 2021 
of dengue cases are asymptomatic and hence the actual numbers of dengue cases are under-reported and many cases are misclassified. One study of the prevalence of dengue estimates that 3.9 billion people in 128 countries are at risk of infection with dengue viruses ${ }^{3}$. The World Health Organization (WHO) ranked dengue ${ }^{4}$ as one of the top ten threats to global health in 2019. All serotypes are not identified in Bangladesh so far. In 2016, a DENV2 outbreak caused around 6,100 clinical cases in Bangladesh $^{5}$. In 2018, clinical incidence of dengue peaked at approximately 6,500 cases, apparently due to circulation of the DENV-3 serotype in the country, which had been notably absent ${ }^{5}$ during 2013-2017. A recently published study ${ }^{6}$ that examined dengue isolates over the three-year period 2015-2017 confirmed co-circulation of DENV-1 and DENV-2 serotypes in Dhaka city, suggesting the absence of DENV-3 and DENV-4. On an average, dengue becomes symptomatic after a 4-to10day incubation period (range, 3-14days). Dengue symptoms usually last 2-7 days. In 2011 revised WHO guidelines, dengue was divided into dengue fever (DF), dengue hemorrhagic fever (DHF) without shock or with shock (DSS) and expanded dengue syndrome ${ }^{7}$. Initial dengue infection may be asymptomatic $(50-90 \%)^{8}$, may result in a nonspecific febrile illness, or may produce the symptom complex of classic dengue fever (DF). Classic dengue fever is marked by rapid onset of high fever, headache, retro-orbital pain, diffuse body pain, weakness, vomiting, sore throat, lymphadenopathy, altered taste sensation, and a centrifugal maculopapular rash among other manifestations. The severity of the pain led to the term breakbone fever to describe dengue. A small percentage of patients develop bleeding and endothelial leak termed severe dengue (DHF and DSS). It is also termed as dengue vasculopathy. Vascular leakage in these patients results in haemoconcentration and serous effusions and can lead to circulatory collapse. This in conjunction with severe haemorrhagic complications, can lead to shock syndrome, which poses a greater fatality risk than bleeding per $\mathrm{se}^{9}$. Expanded dengue syndrome (EDS) was coined by the WHO in the year 2012 to describe cases which do not fall into either dengue shock syndrome or dengue haemorrhagic fever. The atypical manifestations noted in expanded dengue are multi-systemic and multifaceted with organ involvement, such as liver, brain, heart, and kidney ${ }^{10}$. Patients with involvement of gastrointestinal and hepato-biliary system may present with features of asymptomatic elevation of liver enzymes, fulminant hepatic failure, acute pancreatitis, acalculous cholecystitis, peritonitis, sub-acute intestinal obstruction etc.

Laboratory criteria for the diagnosis of dengue include one or more of: detection of virus, viral nucleic acid, antibodies or antigens, or a combination of these. Other relevant investigations may also be required for management of dengue infections.

Dengue fever is usually a self-limited illness. Supportive care with antipyretics like paracetamol, judicious fluid replacement and rest is usually sufficient. Successful management of severe dengue requires intravenous volume replacement, with careful attention to fluid management and proactive treatment of haemorrhage ${ }^{11}$.

Dengue outbreak is not new in Bangladesh, especially in our biggest and capital city, Dhaka. In 2019 (up to 16 September), there were 81,839 dengue cases detected in Bangladesh ${ }^{12}$. But diverse and atypical manifestations of recent dengue outbreak posed a new challenge to us. We have tried to compile all those manifestations and outcome of the study patients that may help in future planning of dengue management.

\section{Methods:}

This study was a hospital based observational study, carried out in Medicine department of Combined Military Hospital (CMH) Dhaka cantonment from May to September 2019. Total 327 dengue patients were enrolled who were admitted to wards of medicine department. Out of 327 patients, as per exclusion criteria 27 were excluded from the study after initial selection and remaining 300 patients were finally selected for the study. Patients aged 11 years or older and only laboratoryconfirmed cases presenting within 7 days of symptom onset were included in the study. The diagnosis was confirmed if at least one of the following criteria was met in acute phase serum: (1) positive dengue-specific non-structural antigen-1 (NS1), (2) positive serology for dengue IgM. Exclusion criteria were patients who presented 7 days after the onset of symptoms, or those who were transferred to other hospitals. Verbal consent was taken and all patients were interviewed and clinical examinations were done. Relevant investigations were done as per requirements of patient management. As this was an observational study and there was no study 
related intervention or invasive procedure, prior ethical clearance was not taken. Data were collected and analyzed by using SPSS 20 and formulated in tables and diagrams.

\section{Results:}

Total 300 patients was studied of whom 11-20 years aged were $37(12.3 \%), 21-40$ years $162(54.0 \%)$, 41-60 years $80(26.7 \%)$ and $>60$ years $21(7.0 \%)$; male were $187(62.3 \%)$ and female $113(37.7 \%)$; service holder $165(55.0 \%)$, housewife $76(25.3 \%)$, retired from service $31(10.3 \%)$, student $18(6.0 \%)$ and business $10(3.3 \%)$ with mean age \pm SD $37.6 \pm 7.5$ years and minimum 12 years, maximum 76 years.

Table-I

\begin{tabular}{lcc}
\multicolumn{3}{c}{ Demographic profile of patients $(N=300)$} \\
Characters & Frequency & Percentage \\
\hline Mean age \pm SD & $37.6 \pm 7.5$ yrs & - \\
Minimum & 12 & - \\
Maximum & 72 & - \\
$11-20$ yrs & 37 & 12.3 \\
$21-40$ yrs & 162 & 54.0 \\
$41-60$ yrs & 80 & 26.7 \\
$>60$ yrs & 21 & 7.0 \\
Gender & & \\
Male & 187 & 62.3 \\
Female & 113 & 37.7 \\
Occupation & & \\
Service & 165 & 55.0 \\
Housewife & 76 & 25.3 \\
Retired from service & 31 & 10.3 \\
Student & 18 & 6.0 \\
Business & 10 & 3.3 \\
\hline
\end{tabular}

Clinical presentation of study patients showed fever 300(100.0\%), headache 265(88.3\%), skin rash 197(65.7\%), bodyache $186(62.0 \%)$, vomiting $152(50.7 \%)$, diarrhoea 65(21.7\%), abdominal pain 58(19.3\%), bleeding manifestation 36(12.0\%), altered sensorium 32(10.7\%), and lymphadenopathy $17(5.7 \%)$ however, most patients had more than one symptom.
Table-II

Clinical presentations of patients $(N=300)$

\begin{tabular}{lcc} 
Presenting complaints & Frequency & Percentage \\
\hline Fever & 300 & 100.0 \\
Headache & 265 & 88.3 \\
Skin rash & 197 & 65.7 \\
Bodyache & 186 & 62.0 \\
Vomiting & 152 & 50.7 \\
Diarrhoea & 65 & 21.7 \\
Abdominal pain & 58 & 19.3 \\
Bleeding manifestation & 36 & 12.0 \\
Altered sensorium & 32 & 10.7 \\
Lymphadenopathy & 17 & 5.7 \\
\hline
\end{tabular}

Among 300 study patients, 84 (28\%) had classical DF, $61(20.3 \%)$ had DHF, 45 (15\%) DSS and $110(36.7 \%)$ had EDS.

Co-morbidity among 300 study patients showed, 126 (42.0\%) had co-morbidity; diabetes mellitus in $37(12.3 \%)$, hypertension in $31(10.3 \%)$, ischemic heart disease in $11(3.7 \%)$, other diseases in $12(4.0 \%)$ and multiple comorbidity in $35(11.7 \%)$.

Table-III

Co-morbidity of study patients $(N=300)$

\begin{tabular}{lcc} 
Diseases & Frequency & Percentage \\
\hline Diabetes mellitus (DM) & 37 & 12.3 \\
Hypertension & 31 & 10.3 \\
Ischemic heart disease (IHD) & 11 & 3.7 \\
Other diseases & 12 & 4.0 \\
Multiple & 35 & 11.7 \\
\hline
\end{tabular}

Laboratory parameters among 300 study patients showed leukopenia 157(52.3\%), leukocytosis 18(6.0\%) and remaining had normal leukocyte count; thrombocytopenia $254(84.7 \%)$ with lowest platelet count $1 \times 10^{9} / \mathrm{L}$; abnormal AST 135 (45.0\%), and abnormal ALT 110 (36.7\%); hyponatraemia 84(28.0\%), hypoalbuminemia 37(12.3\%) and acute kidney injury $43(14.3 \%)$. 
Table-IV

Laboratory parameters of study patients $(N=300)$

\begin{tabular}{lcc} 
Variables & Frequency & Percentage \\
\hline WBC count & 157 & 52.3 \\
Leuckopenia & 18 & 6.0 \\
Leukocytosis & & \\
Platelet count & 254 & 84.7 \\
Thrombocyopenia & $1 \times 10^{9} / \mathrm{L}$ & - \\
Lowest count & 135 & 45.0 \\
AST raised & 110 & 36.7 \\
ALT raised & 84 & 28.0 \\
Hyponatraemia & 37 & 12.3 \\
Hypoalbuminemia & 43 & 14.3 \\
AKI & &
\end{tabular}

\# (AKI, acute Kidney Injury)

Imaging profile of 300 study patients showed,125(41.7\%) patients had pleural effusion of whom 56(18.7\%) right sided, 33(11.0\%) left sided and 36(12.0\%) bilateral; $102(34.0 \%)$ had ascites in ultrasound scan of whom $75(25.0 \%)$ mild, $22(7.3 \%)$ moderate, and $05(1.7 \%)$ severe; $25(8.3 \%)$ patients had acalculous cholecystitis and $07(2.3 \%)$ patients developed cerebrovascular accident in the form of intracranial haemorrhage $5(1.7 \%)$ and $2(0.6 \%)$ infarction.

\section{Table-V}

\section{Imaging profile of study patients $(N=300)$}

\begin{tabular}{lcc} 
Variables & Frequency & Percentage \\
\hline Chest X-ray- & & \\
Pleural effusion & 125 & 41.7 \\
Right & 56 & 18.7 \\
Left & 33 & 11.0 \\
Bilateral & 36 & 12.0 \\
Abdominal USG- & & \\
Ascites present & 102 & 34.0 \\
Mild & 75 & 25.0 \\
Moderate & 22 & 7.3 \\
Severe & 05 & 1.7 \\
Acalculus cholecystitis & 25 & 8.3 \\
Acute pancreatitis & 23 & 7.7 \\
CT/MRI brain- & & \\
CVD & 07 & 2.3 \\
-Intracranial haemorrhage & 5 & 1.7 \\
-Infarction & 2 & 0.6 \\
\hline
\end{tabular}

(\# USG, Ultrasonography; CT, Computed tomography; MRI, Magnetic resonance imaging; CVD, Cerebrovascular disease)
Regarding outcome of study patients showed, cured 295(98.3\%), expired 05(1.7\%); 18(6.0\%) patients needed mechanical ventilator support and $15(5.0 \%)$ required haemodialysis.

\section{Table-VI}

\begin{tabular}{lcc}
\multicolumn{3}{c}{$\begin{array}{r}\text { Outcome and organ support of study } \\
\text { patients }\end{array}$} \\
& $(N=300)$ & \\
Traits & Frequency & Percentage \\
\hline Cured & 295 & 98.3 \\
Expired & 5 & 1.7 \\
Required organ support- & & \\
Mechanical ventilation & 18 & 6.0 \\
Haemodialysis & 15 & 5.0 \\
\hline
\end{tabular}

\section{Discussion:}

Dengue is a highly endemic infectious disease of the tropical countries and is rapidly becoming a global burden. The World Health Organization estimates that 3.9 billion people representing over $40 \%$ of the world's population are at risk of dengue infection ${ }^{13}$. Dengue fever presents in a nonspecific manner similarly to that of many other viral and bacterial illness. Clinical dengue fever typically begins on the third day of illness and persist 5-7days, abating with the cessation of viraemia. Fever may reach $41^{\circ} \mathrm{C}$. Occasionally, and more frequently in children the fever abates for a days and recurs, a pattern that is termed a saddleback fever, which is commonly seen in dengue haemorrhagic fever. In our study, it was found that almost all classes of dengue syndrome and all patients had fever $(300,100 \%)$. Other common presentations were headache $(85.0 \%)$, skin rash $(65.7 \%)$, bodyache $(62.0 \%)$, vomiting $(50.7 \%)$ along with many other symptoms including less common one like lymphadenopathy (5.7\%). In recent years classic dengue fever presentation has expanded its horizon by involving various organ systems which is named as expanded dengue syndrome (EDS). Exact data of Bangladesh regarding expanded dengue syndrome is not known. In the current study EDS was observed in $110(36.7 \%)$ cases similar to very recent scenario observed by Bijaya Mohanty et $\mathrm{al}^{14}$. in their study, $34.8 \%$ (520 out of 1493) cases in a teaching hospital in India. 
Thrombocytopenia is common finding in dengue fever and leukopenia, especially lymphopenia is found near the end of the febrile phase. A platelet count of d" $100,000 / \mathrm{mm}^{3}$ is usually found between the days 3 and 8 of illness. Cytopenias are believed to be caused by direct destructive actions of the virus to bone marrow precursor cells. The resulting active viral replication and cellular destruction in the bone marrow are believed to cause the bone pain ${ }^{11}$. In the current study, leukopenia was observed in $157(51.7 \%)$ cases and thrombocytopenia 254 (84.7\%) cases and few cases of leukocytosis and very few cases of thrombocytosis with no apparent cause. With DENV infection high level of viraemia is associated with involvement of different organs (liver, brain) in the severe form of the disease ${ }^{15}$. Hepatic dysfunction is the crucial feature seen in dengue fever. The pathogenesis of hepatic injury in dengue is believed to be primarily a T-cell mediated process involving interaction between antibodies and the endothelium and a concomitant cytokine storm often labeled as cytokine 'Tsunami,' and host factors like genetic polymorphisms. The spectrum of hepatic involvement includes asymptomatic elevation of hepatic transaminases to occurrence of severe manifestation in the form of acute liver failure ${ }^{16}$. Elevated liver transaminases (AST and ALT) were found in $110(36.7 \%)$ of cases in our study. Liver damage in dengue may also manifests in low albumin levels, and deranged coagulation parameters ${ }^{17}$. Low albumin level was observed in $37(12.3 \%)$ study patients in our study and most patients were from EDS subset.

Plasma leakage is the critical feature of severe dengue. It is caused by increased capillary permeability. Pleural effusion is one of the complications of dengue fever resulting from the plasma leakage into the pleural cavity ${ }^{18}$. Ascites is another manifestation of plasma leakage. Plasma leakage can lead to hypoalbuminemia and shock, which if uncorrected leads to tissue anoxia, metabolic acidosis and death. Bleeding in dengue is caused by capillary fragility and thrombocytopenia and may manifest as petechial skin haemorrhages to life threatening gastrointestinal bleeding ${ }^{11}$. In the current study, pleural effusion was observed in $125(41.7 \%)$ cases, having right sided 56(18.7\%), left sided 33 (11.0\%) and bilateral $36(12.0 \%)$. Shabbir et a ${ }^{19}$. found $12.6 \%$ (10 out of 79) cases of pleural effusion in their study and it was more common in young adult patients than elderly sufferers. It was slightly more common on left side $50 \%$ (5 out of10) as opposed to Wang et al. ${ }^{20}$ who found $62.6 \%$ (92 out of 147) pleural effusion in a study of 363 dengue patients and all pleural effusions were predominantly on the right side $(59.8 \%$; 70 of 117$)$. Our study observed ascites in $102(34.0 \%)$ patients of dengue fever. Acalculous cholecystitis was documented in many case reports of dengue fever. The pathogenesis of acute acalculous cholecystitis is still unclear. It was detected in 25 (22.7\%) EDS patients. A study by Bhatty et al. in 2009 reported $27.5 \%$ of cases as acalculous cholecystitis ${ }^{21}$.In other study ${ }^{14}$ cholecystitis was detected in $21.3 \%$ cases of dengue. Some studies also showed as high as $38 \%$ of cholecystitis in EDS 22 . These patients had an increased levels of alkaline phosphatase, thickened gallbladder wall, and pericholecystic fluid collection. Severe dengue may be complicated by acute pancreatitis. The exact pathogenesis of pancreatic involvement in dengue is not known. But it can be due to result of direct invasion by the virus itself causing inflammation and destruction of pancreatic acinar cells, an autoimmune response to pancreatic islet cells, and development of edema of the ampulla of Vater with obstruction to the outflow of pancreatic fluid ${ }^{23}$. We had $23(7.7 \%)$ dengue cases that developed mild to moderate acute pancreatitis. All cases were from expanded dengue syndrome subset and were managed conservatively.

Acute kidney injury is a serious and potentially lethal complication of severe dengue virus infection and tied to multi-organ failure and increased mortality. Several mechanisms have been proposed for DF-induced acute kidney injury (AKI), including direct action by the virus, hemodynamic instability ${ }^{24}$, rhabdomyolysis, acute glomerular injury by deposition of immune complexes in glomeruli ${ }^{25}$ and hemolytic uremic syndrome ${ }^{26}$. Acute kidney injury was observed in $43(14.3 \%)$ dengue patients in our study of whom $15(5.0 \%)$ patients needed haemodialysis. All patients who required renal replacement therapy had multi-organ dysfunction syndrome (MODS) and needed mechanical ventilator (15 out of 18) support also. In Bangladesh exact incidence of AKI in dengue is not known. Our finding was similar to the incidence of AKI of a study of 620 dengue patients conducted in India (14.51\%, 90 out of $620)^{27}$. Other previous studies ${ }^{28}$ had reported incidence of AKI as $13.3 \%$ and $10.8 \%$ in the same country ${ }^{29}$ in dengue infection, irrespective of severity. 
Neurological manifestations are more commonly observed and reported in dengue involving both central and peripheral nervous system ${ }^{30}$. Dengue patient can present with features of encephalitis, meningitis, stroke, hypokalemic paralysis, encephalopathy, seizures, mononeuropathy, polyneuropathy, and Guillain-Barre syndrome or Miller-Fisher syndromes. Dengue virus infection involving spinal cord is extremely rare. In current study, 7(2.3\%) patients of EDS subset were complicated by cerebrovascular accident of whom intracranial haemorrhage 5(1.7\%) and cerebral infarction 2(0.6\%). Dengue virus infection is associated with significant morbidity and mortality. In previous study ${ }^{31}$ from Bangladesh, case fatality rate of dengue was 1.38\% (195 of 14213) in years 1998-2002 and in 2000-2017 it was 0.66\% ( 268 of 40476 ). In the current study, we observed case fatality $1.7 \%$ (5 of 300) in dengue patients as a whole and $4.5 \%$ (5 of 110) in expanded dengue syndrome cases. All the fatality occurred in expanded dengue syndrome subset of patients and they had multi-organ dysfunction syndrome and needed multiple organ support like haemodialysis $15(5.0 \%)$, mechanical ventilator support $18(6.0 \%)$, or gastrointestinal supportive treatment.

\section{Conclusion:}

A rising tide of expanded dengue syndrome and diverse presentations of dengue syndromes were observed in the study. The occurrence of atypical and systemic presentations in dengue fever may cause increased mortality especially during outbreak. A high degree of clinical suspicion is the key for early diagnosis and treatment and may mitigate adverse outcome of dengue infection. Spreading increased awareness among the community at large is the need of the time to fight against this new challenge.

Conflict of interest:

The authors have no conflict of interest.

\section{References:}

1. Normile D. Surprising new dengue virus throws a spanner in disease control efforts. Science 2013;342(6157):415. https:/ /doi.org/10.1126/science.342.6157.415 PMid:24159024

2. World Health Organization. Dengue and severe dengue. Fact sheet. WHO; 2017.

3. Brady OJ, Gething PW, Bhatt S, Messina JP, Brownstein JS, Hoen AG. Refining the global spatial limits of dengue virus transmission by evidence-based consensus. PLoS Negl Trop Dis 2012;6:e1760. https://doi.org/10.1371/journal.pntd. 0001760 PMid:22880140 PMCid:PMC3413714

4. World Health Organization. Ten threats to global health in 2019. Accessed at: https//www.who.int/ emergencies/ tenthreats-to-global health-in-2019.
5. Muraduzzaman A.K.M., Alam A.N., Sultana S., Siddiqua M., Khan M.H., Akram A. Circulating dengue virus serotypes in Bangladesh from 2013 to 2016. Virus disease. 2018; 29:303-307. https://doi.org/ 10.1007/ s13337-018-0469x PMid:30159364 PMCid:PMC6111961

6. Siddiqua M, Alam AN, Muraduzzaman AKM, Shirin T. NS1 antigen positive dengue infection and molecular characterization of dengue viruses in a private medical college hospital in Dhaka, Bangladesh. Bangladesh J Med Sci 2018; 17: 669-73. https://doi.org/10.3329/bjms. v17i4.38334

7. World Health Organization. Comprehensive guidelines for prevention and control of dengue and dengue hemorrhagic fever (Revised and Expanded). World Health Organization, Regional Office for South-East Asia; 2011.

8. Kyle JL, Harris E. Global spread and persistence of dengue. Annu Rev Microbiol 2008. 62:71-92. https://doi.org/ 10.1146/ annurev.micro. 62.081307.163005 PMid:18429680

9. Statler J, Mammen M, Lyons A, Sun W. Sonographic findings of healthy volunteers infected with dengue virus. J Clin Ultrasound 2008.36(7):413-7. https://doi.org/10.1002/ jcu.20476 PMid:18446859

10. Kadam DB, Salvi S, Chandanwale A. Expanded dengue. J Assoc Physicians India 2016;64:59-63.

11. Smith DS. Dengue: Practice Essentials, Background, Pathophysiology- eMedicine: Medscape:2019; available at https//www. emedicine.medscape.com/dengue.

12. European center for disease prevention and control: Dengue worldwide overview: 2019; accessed at: https// ecdc.europa.eu/dengue.

13. Bhatt S, Gething PW, Brady OJ, Messina JP, Farlow AW, Moyes $\mathrm{CL}$ et al. The global distribution and burden of dengue. Nature 2013;496(7446):504-7. https://doi.org/10.1038/ nature12060 PMid:23563266 PMCid:PMC3651993

14. Bijaya M, Ashok S, Saurabh P. Clinicolaboratory profile of expanded dengue syndrome - Our experience in a teaching hospital: J Fam Med Primary Care 2019;8(3):1022-27. https://doi.org/10.4103/jfmpc.jfmpc_12_19 PMid:31041245 PMCid:PMC6482768

15. Martina BE, Koraka P, Osterhaus AD. Dengue virus pathogenesis: an integrated view. Clin Microbiol Rev 2009;22: 564-81. https://doi.org/10.1128/CMR.00035-09 PMid:19822889 PMCid:PMC2772360

16. Jayanta S, Vishal S. Dengue and its effect on liver. World J Clin Cases 2015;3(2): 125-31. https://doi.org/10.12998/ wjcc.v3.i2.125 PMid:25685758 PMCid:PMC4317605

17. Shah I. Dengue and liver disease. Scand J Infect Dis 2008; 40(11-12):993-4. https://doi.org/10.1080/0036554080 2209027 PMid:18609199

18. Mohamed NA, El-Raoof EA, Ibraheem HA. Respiratory manifestations of dengue fever in Taiz-Yemen. Egypt J Chest Dis Tuberc 2013;62(2):319-23. https://doi.org/ 10.1016/j.ejcdt.2013.03.002 
19. Shabbir M, Ameen F, Roshan N, Israr M. Nature and Clinical Course of Pleural Effusion in Dengue Fever. Int J Intern Emerg Med 2018; 1(1): 1006.

20. Wang CC, Wu CC, Liu JW, Lin AS, Liu SF, Chung YH, et al. Chest radiographic presentation in patients with dengue hemorrhagic fever. Am J Trop Med Hyg 2007;77(2):2916. https://doi.org/10.4269/ajtmh.2007.77.291 PMid: 17690401

21. Bhatty S, Shaikh NA, Fatima M, Sumbhuani AK. Acute acalculous cholecystitis in dengue fever. J Pak Assoc 2009;59:519-21.

22. Chandey M, Kaur H, Kaur S. Acute acalculous cholecystitis in dengue fever patients. Int J Adv Med 2017;4:375-7. https://doi.org/10.18203/2349-3933.ijam20170923

23. Karoli R, Fatima J, Singh G, Maini S. Acute pancreatitis: An unusual complication of dengue fever. J Assoc Physicians India 2012;60:64-5.

24. George R, Liam CK, Chua CT, Lam SK, Pang T, Geethan R, et al. Unusual clinical manifestations of dengue virus infection. Southeast Asian J Trop Med Public Health 1988;19:585-90.

25. Wiwanitkit $\mathrm{V}$. Acute renal failure in the fatal cases of dengue hemorrhagic fever, a summary in Thai death cases. Ren Fail 2005;27:647. https://doi.org/10.1080/08860220500 200916 PMid: 16153008

26. Basu G, Chrispal A, Boorugu H, Gopinath KG, Chandy S, Prakash JA. Acute kidney injury in tropical acute febrile illness in a tertiary care centre - RIFLE criteria validation. Nephrol Dial Transplant 2011;26:524-31. https://doi.org/ 10.1093/ndt/gfq477 PMid:20702532

27. Patel ML, Himanshu D, Chaudhary SC, Atam V, Sachan R, Misra R, Mohapatra SD. Clinical Characteristic and Risk Factors of Acute Kidney Injury among Dengue Viral Infections in Adults: A Retrospective Analysis. Indian J Nephrol 2019;29(1):15-21.

28. Khalil MA, Sarwar S, Chaudry MA, Maqbool B, Khalil Z, Tan J. Acute kidney injury in dengue virus infection. Clin Kidney J 2012;5:390-4. https://doi.org/10.1093/ckj/sfs117 PMid:26019813 PMCid:PMC4432424

29. Mehra N, Patel A, Abraham G, Reddy YN, Reddy YN. Acute kidney injury in dengue fever using Acute Kidney Injury Network criteria: Incidence and risk factors. Trop Doctor 2012; 42:160-2. https://doi.org/10.1258/td.2012. 120023 PMid:22472317

30. Chanthamat N, Sathirapanya P. Acute transverse myelitis associated with dengue viral infection. J Spinal Cord Med 2010;33:425-7. https://doi.org/10.1080/10790268.2010. 11689722 PMid:21061903 PMCid:PMC2964032

31. Mutsuddy P, Jhora ST, Shamsuzzaman A, Kaisar SG, Khan MNA. Dengue situation in Bangladesh: An Epidemiological shift in terms of morbidity and mortality. Can J infect Dis and Med Microbiol 2019; (3516284): 1-12. https://doi.org/ 10.1155/2019/3516284 PMid:30962860 PMCid: PMC 6431455 